\title{
Horseradish peroxidase-labeled silver/reduced graphene oxide thin film-modified screen-printed electrode for detection of carcinoembryonic antigen
}

\begin{abstract}
In this study, a disposable and simple electrochemical immunosensor was fabricated for the detection of carcinoembryonic antigen. In this method, silver nanoparticles (AgNPs) were mixed with reduced graphene oxide (rGO) to modify the surface of screen-printed carbon electrode (SPE). Initially, AgNPs-rGO modified-SPEs were fabricated by using simple electrochemical deposition method. Then the carcinoembryonic antigen (CEA) was immobilized between the primary antibody and horseradish peroxidase (HRP)-conjugated secondary antibody onto AgNPs-rGO modified-SPEs to fabricate a sandwich-type electrochemical immunosensor. The proposed method could detect the CEA with a linear range of $0.05-0.50 \mu \mathrm{g} \mathrm{mL}-1$ and a detection limit down to $0.035 \mu \mathrm{g} \mathrm{mL}-1$ as compared to its non-sandwich counterpart, which yielded a linear range of $0.05-0.40 \mu \mathrm{g} \mathrm{mL}-1$, with a detection limit of $0.042 \mu \mathrm{g} \mathrm{mL}-1$. The immunosensor showed good performance in the detection of carcinoembryonic antigen, exhibiting a simple, rapid and low-cost. The immunosensor showed a higher sensitivity than an enzymeless sensor.
\end{abstract}

Keyword: Silver nanoparticle; Graphene; Carcinoembryonic antigen; Electrochemical sensor; Immunosensor 